\title{
Positive Solutions for a Nonlinear Higher Order Differential System with Coupled Integral Boundary Conditions
}

\author{
Yaohong Li ${ }^{1,2}$ and Haiyan Zhang \\ ${ }^{1}$ School of Mathematics and Statistics, Suzhou University, Anhui 234000, China \\ ${ }^{2}$ School of Mathematics, University of Science and Technology of China, Anhui 230022, China \\ Correspondence should be addressed to Yaohong Li; liyaohon@ustc.edu.cn
}

Received 7 April 2014; Accepted 13 August 2014; Published 24 August 2014

Academic Editor: Yong Shi

Copyright (c) $2014 \mathrm{Y}$. Li and H. Zhang. This is an open access article distributed under the Creative Commons Attribution License, which permits unrestricted use, distribution, and reproduction in any medium, provided the original work is properly cited.

\begin{abstract}
We investigate the existence of positive solutions for a nonlinear higher order differential system, where the differential system is coupled not only in the differential system but also through the boundary conditions. By constructing a special cone and using the fixed point theorem of cone expansion and compression of norm type, the existence of single and multiple positive solutions is established. As an application, we give some examples to demonstrate our results.
\end{abstract}

\section{Introduction}

In this paper, we consider the following nonlinear higher order differential system with coupled integral boundary conditions:

$$
\begin{gathered}
u^{(n)}(t)+a_{1}(t) f_{1}(t, u(t), v(t))=0, \quad t \in(0,1), \\
v^{(n)}(t)+a_{2}(t) f_{2}(t, u(t), v(t))=0, \quad t \in(0,1), \\
u^{(k)}(0)=v^{(k)}(0)=0, \quad k=0,1, \ldots, n-2, \\
u(1)=\alpha[v], \quad v(1)=\beta[u],
\end{gathered}
$$

where $f_{i} \in C([0,1] \times[0,+\infty) \times[0,+\infty),[0,+\infty)), a_{i} \in$ $C([0,1],[0,+\infty)), i=1,2, n \geq 3, \alpha[v], \beta[u]$ are bounded linear functions on $C[0,1]$ given by

$$
\alpha[v]=\int_{0}^{1} v(t) d A(t), \quad \beta[u]=\int_{0}^{1} u(t) d B(t),
$$

involving Stieltjes integrals. In particular, $A, B$ are functionals of bounded variation with positive measures.

In recent years, there were many works to be done for a variety of nonlinear higher order ordinary differential system. However, most papers only focus on paying attention to the differential system with uncoupled boundary conditions (see [1-5] and the reference therein). Coupled boundary conditions arise in the study of reaction-diffusion equations and Sturm-Liouville problems (see [6]) and have wide applications in various fields of sciences and engineering, for example, the heat equation $[7,8]$.

In a recent article [9], by applying a nonlinear alternative of Leray-Schauder type and Guo-Krasnoselskii's fixed point theorem on cone, the authors established the existence of multiple positive solutions of the following system with fourpoint coupled boundary conditions:

$$
\begin{gathered}
D_{0+}^{\alpha} u(t)+\lambda f(t, u(t), v(t))=0, \quad t \in(0,1), \lambda>0, \\
D_{0+}^{\alpha} u(t)+\lambda h(t, u(t), v(t))=0, \quad t \in(0,1), \\
u^{(i)}(0)=v^{(i)}(0)=0, \quad 0 \leq i \leq n-2, \\
u(1)=a v(\xi), \quad v(1)=b u(\eta),
\end{gathered}
$$

where $D_{0+}^{\alpha} u$ is the Riemann-Liouville's fractional derivative.

In [10], by using fixed point index theory, Yang studied the following system with uncoupled boundary conditions:

$$
\begin{gathered}
u^{\prime \prime}(t)+f_{1}(t, u(t), v(t))=0, \quad t \in(0,1), \\
v^{\prime \prime}(t)+f_{2}(t, u(t), v(t))=0, \quad t \in(0,1), \\
u(0)=0, \quad u(1)=\beta[u], \\
v(0)=0, \quad v(1)=\beta[v],
\end{gathered}
$$

where $\beta[\cdot]$ are linear functionals defined by Stieltjes integrals. 
The work of above-mentioned papers and wide applications of coupled boundary value conditions motivate us to study the system (1). Further, the system is coupled not only in the differential system but also through the boundary conditions. By constructing a special cone and using the fixed point theorem on cone expansion and compression, the existence of single and multiple positive solutions is established.

\section{Preliminaries}

Let $E=C[0,1]]$; we write $\|u\|=\max \{|u(t)|: t \in[0,1]\}$. Clearly, $(E,\|\cdot\|)$ is a Banach space. For each $(u, v) \in E \times E$, we write $\|(u, v)\|=\|u\|+\|v\|$. Define

$$
\begin{aligned}
P= & \{u \in E: u(t) \geq 0, t \in[0,1]\}, \\
Q=\{ & (u, v) \in P \times P: \min _{a \leq t \leq b}(u, v) \\
& \left.\stackrel{\text { def }}{=} \min _{a \leq t \leq b}(u(t)+v(t)) \geq \gamma\|(u, v)\|\right\},
\end{aligned}
$$

where $[a, b]$ is some subset of $(0,1), 0<\gamma \leq\left(a^{n-1} \nu / \rho\right)$; consider

$$
\begin{aligned}
& \rho=\max \left\{\frac{\alpha\left[t^{n-1}\right]}{k} \beta[1]+1, \frac{\beta\left[t^{n-1}\right]}{k} \alpha[1]+1,\right. \\
& \left.\frac{\beta[1]}{k}, \frac{\alpha[1]}{k}\right\}, \\
& \nu=\min \left\{\frac{\alpha\left[t^{n-1}\right]}{k} \beta\left[\gamma_{n}(t)\right], \frac{\beta\left[t^{n-1}\right]}{k} \alpha\left[\gamma_{n}(t)\right],\right. \\
& \alpha\left[t^{n-1}\right]=\int_{0}^{1} t^{n-1} d A(t)>0, \\
& \beta\left[t^{n-1}\right]=\int_{0}^{1} t^{n-1} d B(t)>0, \\
& k=1-\alpha\left[t^{n-1}\right] \beta\left[t^{n-1}\right]>0,
\end{aligned}
$$

where $\gamma_{n}(t)$ is defined by the following Lemma 2. Clearly, ( $E \times$ $E,\|\cdot\|)$ is a Banach space and $P$ is a cone of $E$.

Lemma 1. Let $u, v \in E$. Then differential system

$$
\begin{gathered}
u^{(n)}(t)+x(t)=0, \quad t \in(0,1), \\
v^{(n)}(t)+y(t)=0, \quad t \in(0,1), \\
u^{(k)}(0)=v^{(k)}(0)=0, \quad k=0,1, \ldots, n-2, \\
u(1)=\alpha[v], \quad v(1)=\beta[u],
\end{gathered}
$$

has the following integral representation

$$
\begin{aligned}
& u(t)=\int_{0}^{1} F_{1}(t, s) x(s) d s+\int_{0}^{1} G_{1}(t, s) y(s) d s, \\
& v(t)=\int_{0}^{1} F_{2}(t, s) y(s) d s+\int_{0}^{1} G_{2}(t, s) x(s) d s,
\end{aligned}
$$

where

$$
\begin{aligned}
& F_{1}(t, s)=\frac{\alpha\left[t^{n-1}\right] t^{n-1}}{k} \int_{0}^{1} K_{n}(\xi, s) d B(\xi)+K_{n}(t, s), \\
& G_{1}(t, s)=\frac{t^{n-1}}{k} \int_{0}^{1} K_{n}(\xi, s) d A(\xi), \\
& F_{2}(t, s)=\frac{\beta\left[t^{n-1}\right] t^{n-1}}{k} \int_{0}^{1} K_{n}(\xi, s) d A(\xi)+K_{n}(t, s), \\
& G_{2}(t, s)=\frac{t^{n-1}}{k} \int_{0}^{1} K_{n}(\xi, s) d B(\xi), \\
& K_{n}(t, s) \\
& =\frac{1}{(n-1) !}\left\{\begin{array}{l}
t^{n-1}(1-s)^{n-1}-(t-s)^{n-1}, \quad 0 \leq s \leq t \leq 1, \\
t^{n-1}(1-s)^{n-1},
\end{array}\right.
\end{aligned}
$$

Proof. By Taylor's formula, we have

$$
\begin{aligned}
u(t)= & u(0)+t u^{\prime}(0)+\cdots+\frac{t^{n-1}}{(n-1) !} u^{(n-1)}(0) \\
& +\frac{1}{(n-1) !} \int_{0}^{t}(t-s)^{n-1} u^{(n)}(s) d s, \\
v(t)= & v(0)+t v^{\prime}(0)+\cdots+\frac{t^{n-1}}{(n-1) !} v^{(n-1)}(0) \\
& +\frac{1}{(n-1) !} \int_{0}^{t}(t-s)^{n-1} v^{(n)}(s) d s .
\end{aligned}
$$

So, we reduce the equation of problems (7) to the following equivalent integral equation:

$$
\begin{aligned}
& u(t)=-\frac{1}{(n-1) !} \int_{0}^{t}(t-s)^{n-1} x(s) d s+\frac{t^{n-1}}{(n-1) !} u^{(n-1)}(0), \\
& v(t)=-\frac{1}{(n-1) !} \int_{0}^{t}(t-s)^{n-1} y(s) d s+\frac{t^{n-1}}{(n-1) !} v^{(n-1)}(0) .
\end{aligned}
$$

Let $t=1$; we have

$$
\begin{aligned}
& u^{(n-1)}(0)=\int_{0}^{1}(1-s)^{n-1} x(s) d s+(n-1) ! u(1), \\
& v^{(n-1)}(0)=\int_{0}^{1}(1-s)^{n-1} y(s) d s+(n-1) ! v(1) .
\end{aligned}
$$


By substituting $u^{(n-1)}(0)$ and $v^{(n-1)}(0)$ into (12), we have

$$
\begin{aligned}
u(t)= & -\frac{1}{(n-1) !} \int_{0}^{t}(t-s)^{n-1} x(s) d s \\
& +\frac{1}{(n-1) !} \int_{0}^{1} t^{n-1}(1-s)^{n-1} x(s) d s+t^{n-1} u(1) \\
= & \frac{1}{(n-1) !} \int_{0}^{t}\left[t^{n-1}(1-s)^{n-1}-(t-s)^{n-1}\right] x(s) d s \\
& +\frac{1}{(n-1) !} \int_{t}^{1} t^{n-1}(1-s)^{n-1} x(s) d s+t^{n-1} u(1) \\
= & \int_{0}^{1} K_{n}(t, s) x(s) d s+t^{n-1} u(1), \\
v(t)= & \int_{0}^{1} K_{n}(t, s) y(s) d s+t^{n-1} v(1) ;
\end{aligned}
$$

that is,

$$
\begin{aligned}
& u(t)=\int_{0}^{1} K_{n}(t, s) x(s) d s+t^{n-1} u(1) \\
& v(t)=\int_{0}^{1} K_{n}(t, s) y(s) d s+t^{n-1} v(1) .
\end{aligned}
$$

By applying $\beta$ and $\alpha$ to (15), combined with the conditions $u(1)=\alpha[v], v(1)=\beta[u]$, respectively, we obtain

$$
\begin{aligned}
u(1)= & \int_{0}^{1} v(t) d A(t)=\iint_{0}^{1} K_{n}(t, s) y(s) d s d A(t) \\
& +u(1) \int_{0}^{1} t^{n-1} d A(t), \\
v(1)= & \int_{0}^{1} u(t) d B(t)=\iint_{0}^{1} K_{n}(t, s) x(s) d s d B(t) \\
& +u(1) \int_{0}^{1} t^{n-1} d B(t) .
\end{aligned}
$$

Therefore

$$
\begin{aligned}
& \left(\begin{array}{cc}
-\beta\left[t^{n-1}\right] & 1 \\
1 & -\alpha\left[t^{n-1}\right]
\end{array}\right)\left(\begin{array}{l}
u(1) \\
v(1)
\end{array}\right) \\
& =\left(\begin{array}{c}
\iint_{0}^{1} K_{n}(t, s) x(s) d s d B(t) \\
\iint_{0}^{1} K_{n}(t, s) y(s) d s d A(t)
\end{array}\right),
\end{aligned}
$$

and so

$$
\begin{aligned}
\left(\begin{array}{l}
u(1) \\
v(1)
\end{array}\right)= & \frac{1}{k}\left(\begin{array}{cc}
\alpha\left[t^{n-1}\right] & 1 \\
1 & \beta\left[t^{n-1}\right]
\end{array}\right) \\
& \times\left(\begin{array}{c}
\iint_{0}^{1} K_{n}(t, s) x(s) d s d B(t) \\
\iint_{0}^{1} K_{n}(t, s) y(s) d s d A(t)
\end{array}\right) .
\end{aligned}
$$

By substituting (18) into (15), we obtain

$$
\begin{aligned}
u(t)= & \frac{\alpha\left[t^{n-1}\right] t^{n-1}}{k} \iint_{0}^{1} K_{n}(t, s) x(s) d s d B(t) \\
& +\frac{t^{n-1}}{k} \iint_{0}^{1} K_{n}(t, s) y(s) d s d A(t) \\
& +\int_{0}^{1} K_{n}(t, s) x(s) d s, \\
v(t)= & \frac{t^{n-1}}{k} \iint_{0}^{1} K_{n}(t, s) x(s) d s d B(t) \\
& +\frac{\beta\left[t^{n-1}\right] t^{n-1}}{k} \iint_{0}^{1} K_{n}(t, s) y(s) d s d A(t) \\
& +\int_{0}^{1} K_{n}(t, s) y(s) d s .
\end{aligned}
$$

Therefore

$$
\begin{aligned}
u(t)= & \int_{0}^{1}\left[\frac{\alpha\left[t^{n-1}\right] t^{n-1}}{k} \int_{0}^{1} K_{n}(\xi, s) d B(\xi)\right] x(s) d s \\
& +\int_{0}^{1}\left[\frac{t^{n-1}}{k} \int_{0}^{1} K_{n}(\xi, s) d A(\xi)\right] y(s) d s \\
& +\int_{0}^{1} K_{n}(t, s) x(s) d s, \\
v(t)= & \int_{0}^{1}\left[\frac{t^{n-1}}{k} \int_{0}^{1} K_{n}(\xi, s) d B(\xi)\right] x(s) d s \\
& +\int_{0}^{1}\left[\frac{\beta\left[t^{n-1}\right] t^{n-1}}{k} \int_{0}^{1} K_{n}(\xi, s) d A(\xi)\right] y(s) d s \\
& +\int_{0}^{1} K_{n}(t, s) y(s) d s .
\end{aligned}
$$

which is equivalent to system (8).

Lemma 2 (see [11]). The continuous function $K_{n}(t, s)$ has the following properties:

(i) $0 \leq K_{n}(t, s) \leq K_{n}(s)$, for all $t, s \in[0,1]$, where $K_{n}(s)=$ $s(1-s)^{n-1} /(n-2)$ !;

(ii) $K_{n}(t, s) \geq \gamma_{n}(t) K_{n}(s)$, for all $t, s \in[0,1]$, where $\gamma_{n}(t)=$ $(1 /(n-1)) \min \left\{t^{n-1},(1-t) t^{n-2}\right\}$.

Remark 3. By combining (i) and (ii), we can easily see

$$
K_{n}(s) \geq K_{n}(t, s) \geq \gamma_{n}(t) K_{n}(s), \quad \forall t, s \in[0,1] .
$$

Remark 4. From Remark 3, and (9), for $t, s \in[0,1]$, we have

$$
\begin{gathered}
F_{i}(t, s) \leq \rho K_{n}(s), \quad G_{i}(t, s) \leq \rho K_{n}(s), \quad i=1,2, \\
F_{i}(t, s) \geq v t^{n-1} K_{n}(s), \quad G_{i}(t, s) \geq v t^{n-1} K_{n}(s), \quad i=1,2 .
\end{gathered}
$$


Define the operator $T: Q \rightarrow P \times P$ by

$$
T(u, v)=\left(T_{1}(u, v), T_{2}(u, v)\right),
$$

where operators $T_{1}, T_{2}: Q \rightarrow P$ are defined by

$$
\begin{aligned}
T_{1}(u, v)(t)= & \int_{0}^{1} F_{1}(t, s) a_{1}(s) f_{1}(s, u(s), v(s)) d s \\
& +\int_{0}^{1} G_{1}(t, s) a_{2}(s) f_{2}(s, u(s), v(s)) d s, \\
t \in[0,1], & \\
T_{2}(u, v)(t)= & \int_{0}^{1} F_{2}(t, s) a_{2}(s) f_{2}(s, u(s), v(s)) d s \\
& +\int_{0}^{1} G_{2}(t, s) a_{1}(s) f_{1}(s, u(s), v(s)) d s, \\
& t \in[0,1] .
\end{aligned}
$$

Moreover, by Lemma 1 , if $(u, v) \in Q$ is a fixed point of the operator $T$, then $(u, v)$ is a solution of the system (1).

Lemma 5. The operator $T: Q \rightarrow Q$ is completely continuous.

Proof. By Remark 4, for $s \in[0,1]$, we obtain

$$
\begin{aligned}
& \min _{t \in[a, b]} F_{i}(t, s) \geq v a^{n-1} K_{n}(s), \\
& \min _{t \in[a, b]} G_{i}(t, s) \geq v a^{n-1} K_{n}(s), \quad i=1,2 .
\end{aligned}
$$

Therefore, By Lemma 2 and Remark 3, for $(u, v) \in P$, we have

$$
\begin{aligned}
\left\|T_{1}(u, v)\right\| \leq & \rho \int_{0}^{1} K_{n}(s) a_{1}(s) f_{1}(s, u(s), v(s)) d s \\
& +\rho \int_{0}^{1} K_{n}(s) a_{2}(s) f_{2}(s, u(s), v(s)) d s .
\end{aligned}
$$

Moreover, we have

$$
\begin{aligned}
& \min _{t \in[a, b]} T_{1}(u, v)(t) \\
& =\min _{t \in[a, b]}\left[\int_{0}^{1} F_{1}(t, s) a_{1}(s) f_{1}(s, u(s), v(s)) d s\right. \\
& \left.\quad+\int_{0}^{1} G_{1}(t, s) a_{2}(s) f_{2}(s, u(s), v(s)) d s\right] \\
& \geq v a^{n-1}\left[\int_{0}^{1} K_{n}(s) a_{1}(s) f_{1}(s, u(s), v(s)) d s\right. \\
& \left.\quad+\int_{0}^{1} K_{n}(s) a_{2}(s) f_{2}(s, u(s), v(s)) d s\right] \\
& \geq \gamma\left\|T_{1}(u, v)\right\| .
\end{aligned}
$$

In the same way, we can prove that

$$
\min _{t \in[a, b]} T_{2}(u, v)(t) \geq \gamma\left\|T_{2}(u, v)\right\| .
$$

Thus

$$
\begin{aligned}
\min _{t \in[a, b]} T(u, v) & =\min _{t \in[a, b]}\left(T_{1}(u, v)(t)+T_{2}(u, v)(t)\right) \\
& \geq \gamma\left\|T_{1}(u, v)\right\|+\gamma\left\|T_{2}(u, v)\right\|=\gamma\|T(u, v)\|
\end{aligned}
$$

Then operator $T: Q \rightarrow Q$ is continuous since $K_{n}(t, s)$, $f_{1}(t, u, v), f_{2}(t, u, v), a_{1}(t), a_{2}(t)$ are continuous. Standard applications of Arzelà-Ascoli theorem; it is easy to prove that operator $T: Q \rightarrow Q$ is completely continuous.

Lemma 6 (see [12]). Suppose $E$ is a real Banach space and $P$ is cone in $E$, and let $\Omega_{1}, \Omega_{2}$ be bounded open sets in $E$ such that $\theta \in \Omega_{1}, \bar{\Omega}_{1} \subset \Omega_{2}$. Let operator $T: P \cap\left(\bar{\Omega}_{2} \backslash \Omega_{1}\right) \rightarrow P$ be completely continuous. Suppose that one of the following two conditions holds:

(i) $\|T u\| \leq\|u\|$, for all $u \in P \cap \partial \Omega_{1} ;\|T u\| \geq\|u\|$, for all $u \in P \cap \partial \Omega_{2}$,

(ii) $\|T u\| \geq\|u\|$, for all $u \in P \cap \partial \Omega_{1} ;\|T u\| \leq\|u\|$, for all $u \in P \cap \partial \Omega_{2}$,

then operator $T$ has at least one fixed point in $P \cap\left(\bar{\Omega}_{2} \backslash \Omega_{1}\right)$.

\section{Main Results}

In this section, we show the existence of positive solutions to the system (1). For convenience, we first introduce the following notations:

$$
\begin{aligned}
& f_{1 \lambda}=\liminf _{u+v \rightarrow \lambda} \min _{t \in[0,1]} \frac{f_{1}(t, u, v)}{u+v}, \\
& f^{1 \lambda}=\limsup _{u+v \rightarrow \lambda} \max _{t \in[0,1]} \frac{f_{1}(t, u, v)}{u+v}, \\
& f_{2 \lambda}=\liminf _{u+v \rightarrow \lambda} \min _{t \in[0,1]} \frac{f_{2}(t, u, v)}{u+v}, \\
& f^{2 \lambda}=\limsup _{u+v \rightarrow \lambda} \max _{t \in[0,1]} \frac{f_{2}(t, u, v)}{u+v},
\end{aligned}
$$

where $\lambda=0$ or $\infty$. Let $r=\min \left\{r_{1}, r_{2}\right\}, R=\max \left\{R_{1}, R_{2}\right\}$, where

$$
\begin{array}{r}
r_{i}=\left(4 \rho \int_{0}^{1} a_{i}(s) K_{n}(s) d s\right)^{-1}, \\
R_{i}=\left(4 \gamma v a^{n-1} \int_{a}^{b} a_{i}(s) K_{n}(s) d s\right)^{-1}, \\
i=1,2 .
\end{array}
$$

Theorem 7. If $f^{10}, f^{20} \in[0, r)$ and $f_{1 \infty}, f_{2 \infty} \in(R,+\infty]$, then system (1) has at least one positive solution. 
Proof. At first, it follows from the assumption $f^{10}, f^{20} \in[0, r)$ that there exists $\mu_{1}>0$ and a sufficiently small $\varepsilon_{1}>0$ such that

$$
\begin{array}{ll}
f_{1}(t, u, v) \leq\left(f^{10}+\varepsilon_{1}\right)(u+v), & \forall t \in[0,1], u+v \leq \mu_{1}, \\
f_{2}(t, u, v) \leq\left(f^{20}+\varepsilon_{1}\right)(u+v), & \forall t \in[0,1], u+v \leq \mu_{1},
\end{array}
$$

where $\varepsilon_{1}$ satisfies $f^{10}+\varepsilon_{1} \leq r$ and $f^{20}+\varepsilon_{1} \leq r$.

Set $\Omega_{1}=\left\{(u, v) \in P \times P:\|(u, v)\|<\mu_{1}\right\}$. For any $(u, v) \in$ $\partial \Omega_{1} \cap Q$, by (24), (25), and (33), we have

$$
\begin{aligned}
\left\|T_{1}(u, v)\right\| \leq & \rho \int_{0}^{1} K_{n}(s) a_{1}(s) f_{1}(s, u(s), v(s)) d s \\
& +\rho \int_{0}^{1} K_{n}(s) a_{2}(s) f_{2}(s, u(s), v(s)) d s \\
\leq & \rho\left[\left(f^{10}+\varepsilon_{1}\right) \int_{0}^{1} K_{n}(s) a_{1}(s) d s\right. \\
& \left.+\left(f^{20}+\varepsilon_{1}\right) \int_{0}^{1} K_{n}(s) a_{2}(s) d s\right] \cdot\|(u, v)\| \\
\leq & \rho\left[\frac{1}{4 \rho}+\frac{1}{4 \rho}\right]\|(u, v)\| \leq \frac{1}{2}\|(u, v)\|, \\
\left\|T_{2}(u, v)\right\| \leq & \rho \int_{0}^{1} K_{n}(s) a_{2}(s) f_{2}(s, u(s), v(s)) d s \\
& +\rho \int_{0}^{1} K_{n}(s) a_{1}(s) f_{1}(s, u(s), v(s)) d s \\
\leq & \rho\left[\left(f^{20}+\varepsilon_{1}\right) \int_{0}^{1} K_{n}(s) a_{2}(s) d s\right. \\
& \left.+\left(f^{10}+\varepsilon_{1}\right) \int_{0}^{1} K_{n}(s) a_{1}(s) d s\right] \cdot\|(u, v)\| \\
\leq & \rho\left[\frac{1}{4 \rho}+\frac{1}{4 \rho}\right]\|(u, v)\| \leq \frac{1}{2}\|(u, v)\| .
\end{aligned}
$$

Therefore

$$
\begin{array}{r}
\|T(u, v)\|=\left\|T_{1}(u, v)\right\|+\left\|T_{2}(u, v)\right\| \leq\|(u, v)\|, \\
\text { for }(u, v) \in \partial \Omega_{1} \cap Q .
\end{array}
$$

Further, it follows from the the assumption $f_{1 \infty}, f_{2 \infty} \in$ $(R,+\infty]$ that there exists $l>\mu_{1}>0$ and a sufficiently small $\varepsilon_{2}>0$ such that

$$
\begin{array}{ll}
f_{1}(t, u, v) \geq\left(f_{10}-\varepsilon_{2}\right)(u+v), & \forall t \in[0,1], u+v \geq l, \\
f_{2}(t, u, v) \geq\left(f_{2 \infty}-\varepsilon_{2}\right)(u+v), & \forall t \in[0,1], u+v \geq l,
\end{array}
$$

where $\varepsilon_{2}$ satisfies $f_{1 \infty}-\varepsilon_{2} \geq R$ and $f_{2 \infty}-\varepsilon_{2} \geq R$. Let $\mu_{2}=$ $\left.\max \left\{2 \mu_{1}, l / \gamma\right\}\right\}$; set $\Omega_{2}=\left\{(u, v) \in P \times P:\|(u, v)\|<\mu_{2}\right\}$. Then $(u, v) \in \partial \Omega_{2} \cap Q$ implies that $\min _{t \in[a, b]}(u, v) \geq \gamma\|(u, v)\|=$ $\gamma \mu_{2} \geq l$. So, by (24), (25), and (36), we have

$$
\begin{gathered}
\min _{t \in[a, b]} T_{1}(u, v)(t) \\
\geq v a^{n-1}\left[\int_{a}^{b} K_{n}(s) a_{1}(s) f_{1}(s, u(s), v(s)) d s\right. \\
\left.\quad+\int_{a}^{b} K_{n}(s) a_{2}(s) f_{2}(s, u(s), v(s)) d s\right] \\
\geq \gamma v a^{n-1}\left[\left(f_{1 \infty}-\varepsilon_{2}\right) \int_{a}^{b} K_{n}(s) a_{1}(s) d s\right. \\
\left.\quad+\left(f_{2 \infty}-\varepsilon_{2}\right) \int_{a}^{b} K_{n}(s) a_{2}(s) d s\right] \cdot\|(u, v)\| \\
\geq \gamma v a^{n-1}\left[\frac{1}{4 \gamma v a^{n-1}}+\frac{1}{4 \gamma v a^{n-1}}\right]\|(u, v)\| \\
\geq \frac{1}{2}\|(u, v)\|, \\
\min _{t \in[a, b]} T_{2}(u, v)(t) \\
\geq v a^{n-1}\left[\int_{a}^{b} K_{n}(s) a_{2}(s) f_{2}(s, u(s), v(s)) d s\right. \\
\left.\quad+\int_{a}^{b} K_{n}(s) a_{1}(s) f_{1}(s, u(s), v(s)) d s\right] \\
\geq \frac{1}{2}\|(u, v)\| . \\
\left.\quad+\left(f_{1 \infty}-\varepsilon_{1}\right) \int_{a}^{b} K_{n}(s) a_{1}(s) d s\right] \cdot\|(u, v)\| \\
{\left[\left(f_{2 \infty}-\varepsilon_{2}\right) \int_{a}^{b} K_{n}(s) a_{2}(s) d s\right.}
\end{gathered}
$$

Therefore,

$$
\begin{aligned}
\|T(u, v)\| & =\left\|T_{1}(u, v)\right\|+\left\|T_{2}(u, v)\right\| \\
& \geq \min _{t \in[a, b]} T_{1}(u, v)(t)+\min _{t \in[a, b]} T_{2}(u, v)(t) \\
& \geq\|(u, v)\|, \quad(u, v) \in \partial \Omega_{2} \cap Q .
\end{aligned}
$$

By applying Lemmas 5 and 6 to (35) and (38), it follows that operator $T$ has at least one fixed point $(u, v)$ in $Q \cap\left(\bar{\Omega}_{2} \backslash \Omega_{1}\right)$. This means that system (1) has at least one positive solution $(u, v)$.

Using similar arguments as those used in the proof of Theorem 7, we can also obtain the following result.

Theorem 8. If $f^{1 \infty}, f^{2 \infty} \in[0, r)$ and $f_{10}, f_{20} \in(R,+\infty]$, then system (1) has at least one positive solution. 
Next we discuss the multiplicity of positive solutions for system (1).

Theorem 9. If $f_{10}, f_{2 \infty} \in(4 R,+\infty]$, and there exist an $m>$ 0 such that $f_{1}(t, u, v), f_{2}(t, u, v) \in(0, m r)$, for for all $t \in$ $[0,1],(u, v) \in \partial \Omega_{3} \cap Q$, where $\Omega_{3}=\{(u, v) \in P \times P,\|(u, v)\|<$ $m\}$, then system (1) has at least two positive solutions.

Proof. At first, it follows from the assumption $f_{10} \in$ $(4 R,+\infty],+\infty]$ that there exists an $0<m_{1}<m$ and a sufficiently small $\varepsilon_{4}>0$ such that

$$
f_{1}(t, u, v) \geq\left(f_{10}-\varepsilon_{4}\right)(u+v), \quad \forall t \in[0,1], u+v \leq m_{1},
$$

where $\varepsilon_{4}$ satisfies $f_{10}-\varepsilon_{4} \geq 4 R$.

Set $\Omega_{4}=\left\{(u, v) \in P \times P:\|(u, v)\|<m_{1}\right\}$ and $(u, v) \in$ $\partial \Omega_{4} \cap Q$. By (24), we have

$$
\begin{aligned}
\|T(u, v)\| & \geq \min _{t \in[a, b]} T(u, v)(t) \geq \min _{t \in[a, b]} T_{1}(u, v)(t) \\
& \geq v a^{n-1} \int_{a}^{b} K_{n}(s) a_{1}(s) f_{1}(s, u(s), v(s)) d s \\
& \geq \gamma v a^{n-1}\left(f_{10}-\varepsilon_{4}\right) \int_{a}^{b} K_{n}(s) a_{1}(s) d s \cdot\|(u, v)\| \\
& \geq\|(u, v)\| .
\end{aligned}
$$

Further, by using $f_{2 \infty} \in(4 R,+\infty]$, there exists $m_{2}>m>0$ and a sufficiently small $\varepsilon_{5}>0$ such that

$$
f_{2}(t, u, v) \geq\left(f_{2 \infty}-\varepsilon_{5}\right)(u+v), \quad \forall t \in[0,1], u+v \geq m_{2},
$$

where $\varepsilon_{5}$ satisfies $f_{2 \infty}-\varepsilon_{5} \geq 4 R$. Set $\Omega_{5}=\{(u, v) \in P \times P$ : $\left.\|(u, v)\|<m_{3}\right\}$, where $m_{3}>m_{2}$. For all $(u, v) \in \partial \Omega_{5} \cap Q$, by (24), we have

$$
\begin{aligned}
\|T(u, v)\| & \geq \min _{t \in[a, b]} T(u, v)(t) \geq \min _{t \in[a, b]} T_{1}(u, v)(t) \\
& \geq v a^{n-1} \int_{a}^{b} K_{n}(s) a_{2}(s) f_{2}(s, u(s), v(s)) d s \\
& \geq \gamma v a^{n-1}\left(f_{2 \infty}-\varepsilon_{5}\right) \int_{a}^{b} K_{n}(s) a_{2}(s) d s \cdot\|(u, v)\| \\
& \geq\|(u, v)\| .
\end{aligned}
$$

By assumption, for for all $(u, v) \in \partial \Omega_{3} \cap Q$, we have

$$
\begin{aligned}
\|T(u, v)\| & =\left\|T_{1}(u, v)\right\|+\left\|T_{2}(u, v)\right\| \\
& \leq 2 r m \rho\left[\int_{0}^{1} K_{n}(s) a_{1}(s) d s+\int_{0}^{1} K_{n}(s) a_{2}(s) d s\right] \\
& \leq m=\|(u, v)\| .
\end{aligned}
$$

From (40)-(43), it is easy to know that two conditions of Lemma 6 are both satisfied. By applying Lemmas 5 and 6 to (40)-(43), it follows that operator $T$ has at least a fixed point $\left(u_{1}, v_{1}\right) \in Q \cap\left(\bar{\Omega}_{3} \backslash \Omega_{4}\right)$ and a fixed point $\left(u_{2}, v_{2}\right) \in$ $Q \cap\left(\bar{\Omega}_{5} \backslash \Omega_{3}\right)$. Both are positive solutions of system (1) and satisfy $m_{1} \leq\left\|\left(u_{1}, v_{1}\right)\right\|<m<\left\|\left(u_{2}, v_{2}\right)\right\| \leq m_{3}$. This means system (1) has at least two positive solutions.

Similarly, we have the following results.

Theorem 10. If $f_{10}, f_{1 \infty} \in(4 R,+\infty]$, and there exist an $m>0$ such that $f_{1}(t, u, v), f_{2}(t, u, v) \in(0, m r)$, for for all $t \in$ $[0,1],(u, v) \in \partial \Omega_{6} \cap Q$, where $\Omega_{6}=\{(u, v) \in P \times P,\|(u, v)\|<$ $m\}$, then system (1) has at least two positive solutions.

Theorem 11. If $f_{20}, f_{1 \infty} \in(4 R,+\infty]$, and there exist an $m>0$ such that $f_{1}(t, u, v), f_{2}(t, u, v) \in(0, m r)$, for all $t \in$ $[0,1],(u, v) \in \partial \Omega_{7} \cap Q$, where $\Omega_{7}=\{(u, v) \in P \times P,\|(u, v)\|<$ $m\}$. Then system (1) has at least two positive solutions.

Theorem 12. If $f_{20}, f_{2 \infty} \in(4 R,+\infty]$, and there exist an $m>0$ such that $f_{1}(t, u, v), f_{2}(t, u, v) \in(0, m r)$, for all $t \in$ $[0,1],(u, v) \in \partial \Omega_{8} \cap Q$, where $\Omega_{8}=\{(u, v) \in P \times P,\|(u, v)\|<$ $m$ \}. Then system (1) has at least two positive solutions.

\section{Some Examples}

In order to illustrate our result, we consider some examples.

Example 1. Consider the following system

$$
\begin{aligned}
& -u^{(3)}(t)=\frac{1+t}{8}\left[\left(u^{2}+v^{2}\right)+\lambda \sin (u+v)\right], \quad t \in(0,1) \\
& -v^{(3)}(t)=\frac{1}{4}\left[\left(u^{2}+v^{2}\right)^{2}+e^{-\left(u^{2}+v^{2}\right)}\right], \quad t \in(0,1) \\
& u(0)=u^{\prime}(0)=0, \quad u(1)=2 \int_{0}^{1} v(t) d t \\
& v(0)=v^{\prime}(0)=0, \quad v(1)=\int_{0}^{1} u(t) d t
\end{aligned}
$$

where $n=3, a_{1}(t)=(1+t) / 8, a_{2}(t)=1 / 4, f_{1}(t, u, v)=$ $\left(u^{2}+v^{2}\right)+\lambda \sin (u+v), f_{2}(t, u, v)=\left(u^{2}+v^{2}\right)^{2}+e^{-\left(u^{2}+v^{2}\right)}$, $A(t)=2 t, B(t)=t, K_{n}(s)=s(1-s)^{2}, \gamma_{n}(t)=(1 / 2) \min \left\{t^{2},(1-\right.$ $t) t\}$. By direct calculation, we can obtain that $\rho=18 / 7, v=$ $3 / 56, r=14 / 15$. Choose $\lambda \in[0,14 / 15))$; then conditions of Theorem 7 are satisfied. This means that system (44) has at least one position solution.

Example 2. Consider the following system

$$
\begin{aligned}
& -u^{(3)}(t)=\frac{1+t}{16}\left(u^{2}+v^{2}\right)^{(1 / 3)}, \quad t \in(0,1), \\
& -v^{(3)}(t)=\frac{1}{32}\left(u^{2}+v^{2}\right), \quad t \in(0,1),
\end{aligned}
$$




$$
\begin{array}{ll}
u(0)=u^{\prime}(0)=0, & u(1)=2 \int_{0}^{1} v(t) d t, \\
v(0)=v^{\prime}(0)=0, & v(1)=\int_{0}^{1} u(t) d t
\end{array}
$$

where $n=3, a_{1}(t)=(1+t) / 16, a_{2}(t)=1 / 32, f_{1}(t, u, v)=$ $\left(u^{2}+v^{2}\right)^{1 / 3}, f_{2}(t, u, v)=\left(u^{2}+v^{2}\right), A(t)=2 t, B(t)=$ $t, K_{n}(s)=s(1-s)^{2}, \gamma_{n}(t)=(1 / 2) \min \left\{t^{2},(1-t) t\right\}$. By direct calculation, we can obtain that $\rho=(18 / 7), v=(3 / 56), \backslash, r=$ (112/15). Choose $m=2$; then conditions of Theorem 9 are satisfied. This means that system (45) has at least two position solutions.

\section{Conflict of Interests}

The authors declare that there is no conflict of interests regarding the publication of this paper.

\section{Authors' Contribution}

The work presented here was carried out in collaboration between all authors. All authors read and approved the final paper.

\section{Acknowledgments}

The authors are grateful to the referees for their careful reading. This research is supported by the Nature Science Foundation of Anhui Provincial Education Department (Grant nos. KJ2014A252 and KJ2013A248) and Professors (Doctors) Scientific Research Foundation of Suzhou University (Grant no. 2013jb04).

\section{References}

[1] J. Henderson and R. Luca, "Positive solutions for a system of higher-order multi-point boundary value problems," Computers \& Mathematics with Applications, vol. 62, no. 10, pp. 39203932, 2011.

[2] S. Xie and J. Zhu, "Positive solutions of the system for $n$ th-order singular nonlocal boundary value problems," Journal of Applied Mathematics and Computing, vol. 37, no. 1-2, pp. 119-132, 2011.

[3] J. Henderson and R. Luca, "On a system of higher-order multi-point boundary value problems," Electronic Journal of Qualitative Theory of Differential Equations, vol. 2012, no. 49, pp. 1-14, 2012.

[4] J. Xu and Z. Yang, "Positive solutions for a system of nth order nonlinear boundary value problems," Electronic Journal of Qualitative Theory of Differential Equations, vol. 2012, no. 4, pp. 1-16, 2012.

[5] X. Zhang, C. Zhu, and Z. Wu, "Solvability for a coupled system of fractional differential equations with impulses at resonance," Boundary Value Problems, vol. 2013, article 80, 2013.

[6] H. Amann, "Parabolic evolution equations and nonlinear boundary conditions," Journal of Differential Equations, vol. 72, no. 2, pp. 201-269, 1988.
[7] Z. Lin and C. Xie, "The blow-up rate for a system of heat equations with nonlinear boundary conditions," Nonlinear Analysis, vol. 34, no. 5, pp. 767-778, 1998.

[8] M. Pedersen and Z. Lin, "Blow-up analysis for a system of heat equations coupled through a nonlinear boundary condition," Applied Mathematics Letters, vol. 14, no. 2, pp. 171-176, 2001.

[9] C. Yuan, D. Jiang, D. O'Regan, and R. P. Agarwal, "Multiple positive solutions to systems of nonlinear semipositone fractional differential equations with coupled boundary conditions," Electronic Journal of Qualitative Theory of Differential Equations, vol. 2012, no. 13, pp. 1-17, 2012.

[10] Z. Yang, "Positive solutions to a system of second-order nonlocal boundary value problems," Nonlinear Analysis: Theory, Methods \& Applications, vol. 62, no. 7, pp. 1251-1265, 2005.

[11] D. Xie, C. Bai, Y. Liu, and C. Wang, "Positive solutions for nonlinear semipositone nth-order boundary value problem," Electronic Journal of Qualitative Theory of Differential Equations, vol. 2008, no. 7, pp. 1-12, 2008.

[12] D. J. Guo and V. Lakshmikantham, Nonlinear Problems in Abstract Cones, vol. 5, Academic Press, San Diego, Calif, USA, 1988. 


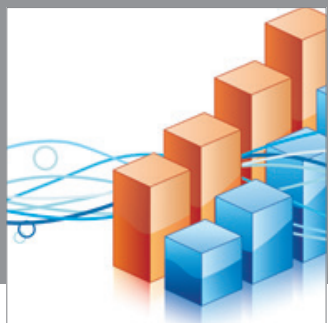

Advances in

Operations Research

mansans

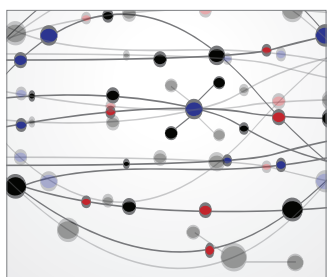

The Scientific World Journal
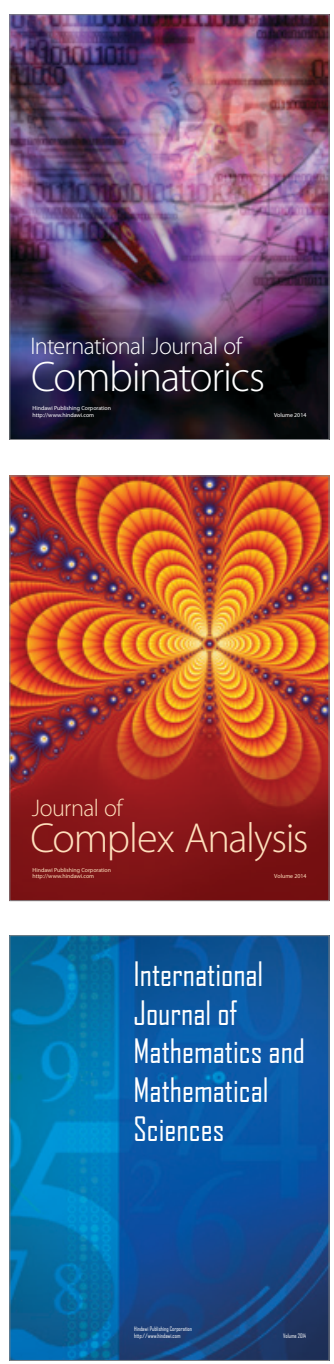
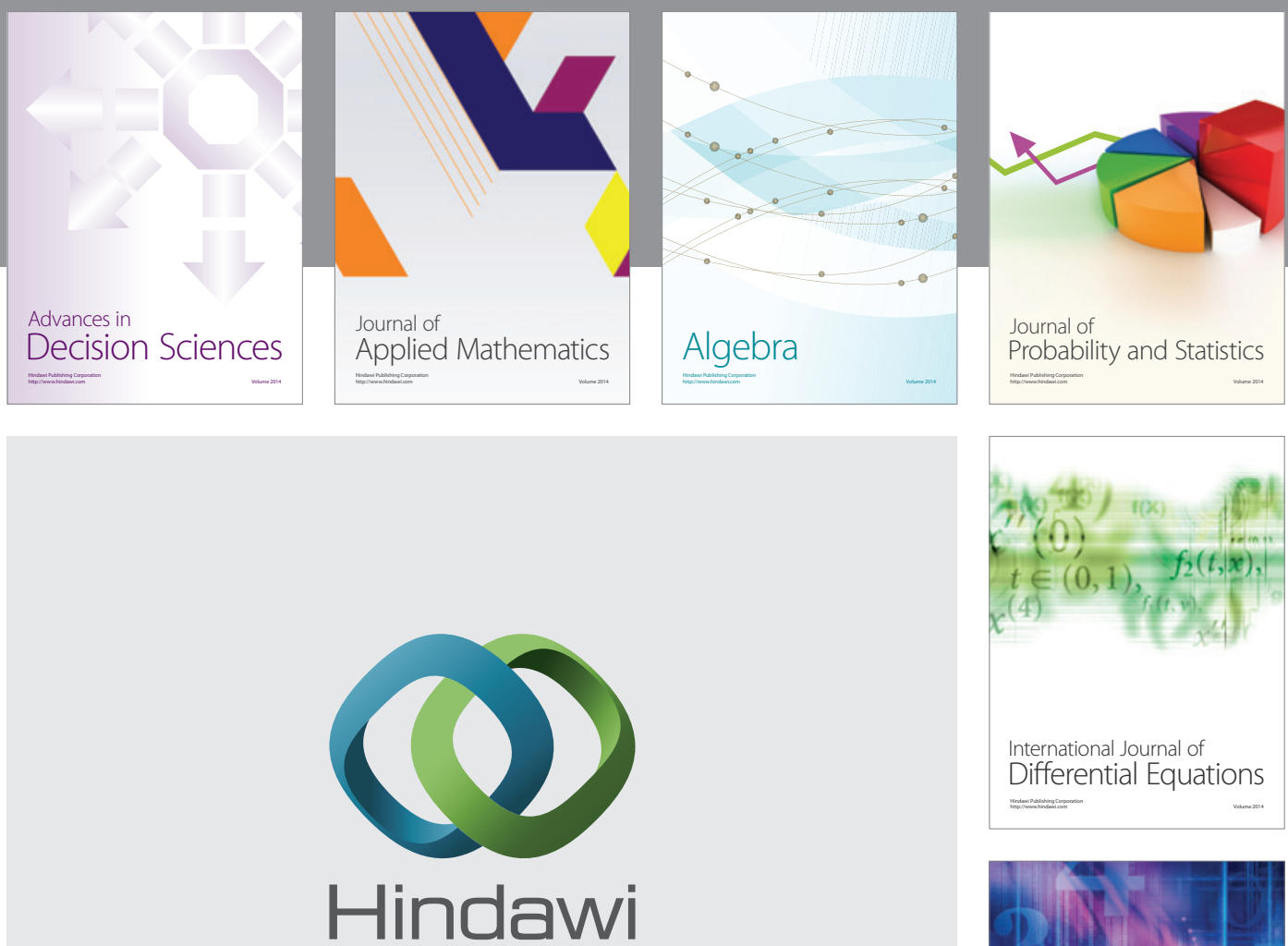

Submit your manuscripts at http://www.hindawi.com
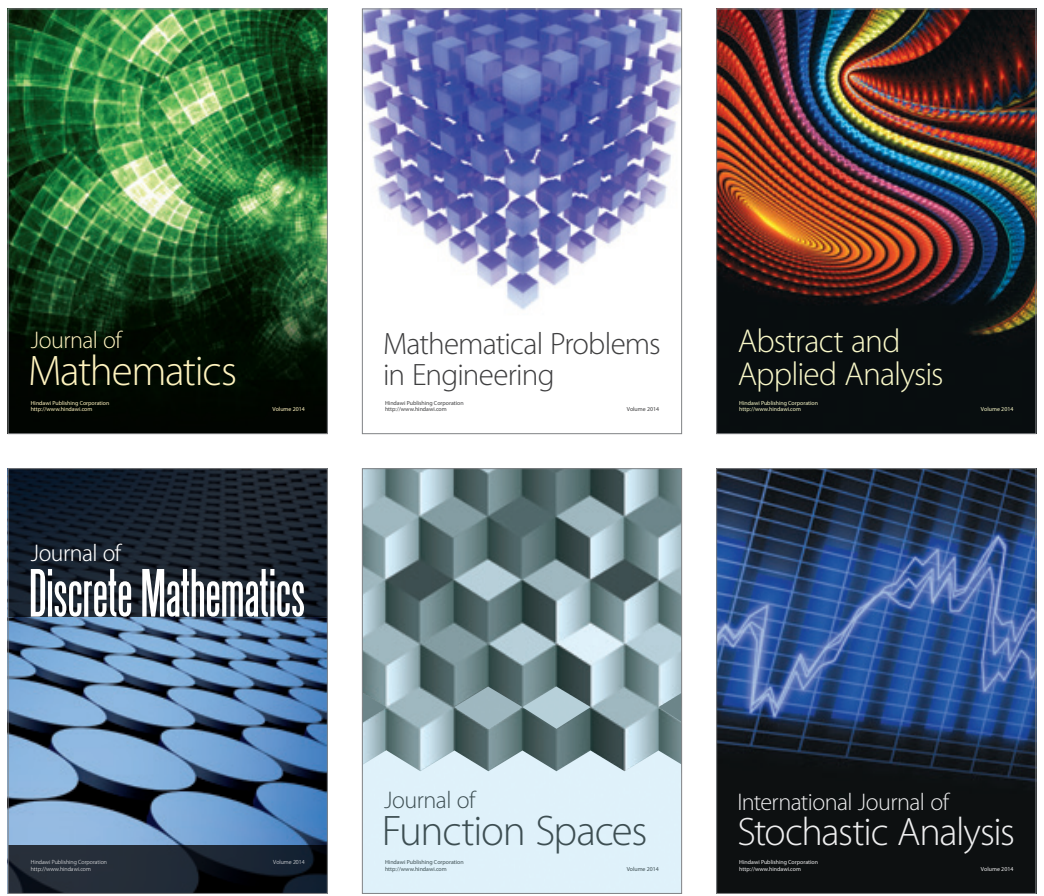

Journal of

Function Spaces

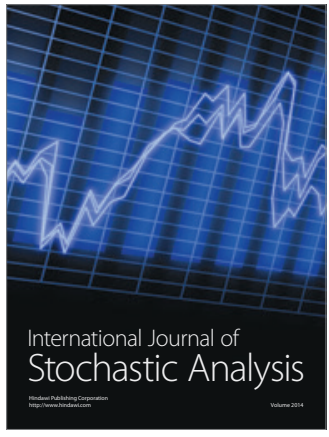

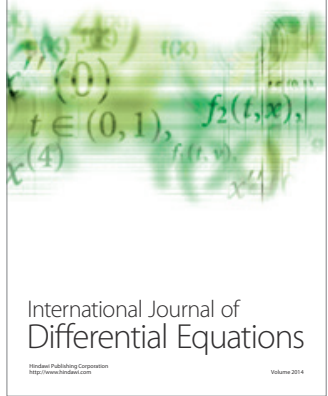
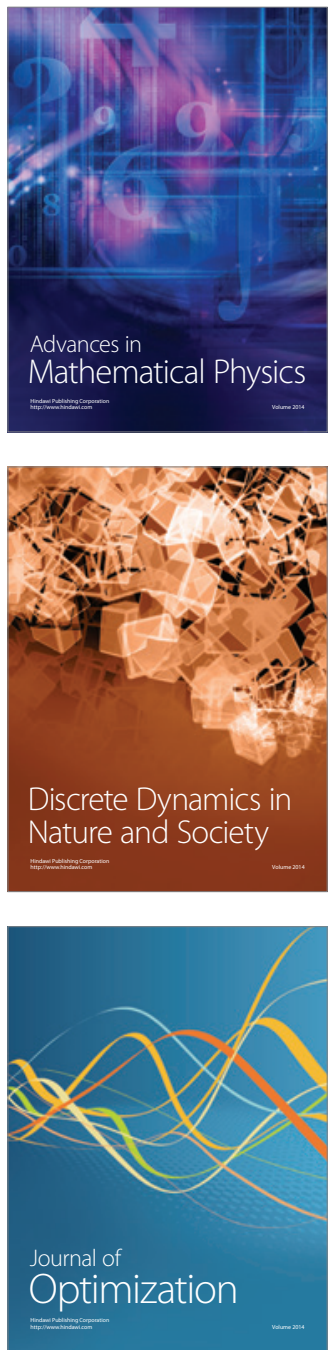